\title{
BMJ Cardiac arrest management in general Open practice in Ireland: a 5-year cross-sectional study
}

\author{
G Bury, ${ }^{1}$ M Headon, ${ }^{1}$ M Egan, ${ }^{1}$ J Dowling ${ }^{2}$
}

To cite: Bury G, Headon M, Egan $\mathrm{M}$, et al. Cardiac arrest management in general practice in Ireland: a 5-year cross-sectional study. BMJ Open 2013;3:e002563. doi:10.1136/bmjopen-2013002563

- Prepublication history for this paper are available online. To view these files please visit the journal online (http://dx.doi.org/10.1136/ bmjopen-2013-002563).

Received 4 January 2013 Revised 12 April 2013 Accepted 15 April 2013

This final article is available for use under the terms of the Creative Commons Attribution Non-Commercial 2.0 Licence; see http://bmjopen.bmj.com

${ }^{1}$ Centre for Emergency Medical Science, School of Medicine \& Medical Science, University College Dublin, Dublin, Ireland

${ }^{2}$ Northwest Immediate Care Programme,

Manorcunningham, County Donegal, Ireland

Correspondence to Dr G Bury; gerard.bury@ucd. ie

\section{ABSTRACT}

Objective: To document the involvement of general practitioners (GPs) in cardiac arrests with resuscitation attempts (CARAs) and to describe the outcomes.

Design: A 5-year prospective cross-sectional study of GPs in Ireland equipped with automated external defibrillators (AEDs) and immediate care training by the MERIT Project, with data collection every 3 months over the 5 -year period. Practices reported CARAs by quarterly survey with an $89 \%$ mean response rate (81-97\% for the period).

Setting: General practices throughout Ireland. Participants: 495 GP participated: 168 (33.9\%) urban, $163(32.9 \%)$ rural and $164(33.1 \%)$ mixed. Interventions: All participating practices received a standard AED and basic life support kit. Training in immediate care was provided for at least one GP in the practice.

Main outcome measures: Incidence of CARA in participating practices. Return of spontaneous circulation (ROSC) and discharge alive from hospital.

Results: $36 \%$ of practices were involved in a CARA during the 5 -year period and $13 \%$ were involved in more than one CARA. Of the 272 CARAs reported, ROSC occurred in $32 \%$ (87/272) and discharge from hospital in $18.7 \%$ (49/262). In $45 \%$ of cases, the first AED was brought by the GP and in $65 \%$, the GP arrived before the ambulance service. More cases occurred in rural and mixed settings than urban ones, but the survival rates did not differ between areas. In $65 \%$ of cases, the GP was on duty at the time of the incident and $47 \%$ of cases occurred in the patient's home.

Conclusions: These outcomes are comparable with more highly structured components of the emergency response system and indicate that GPs have an important role to play in the care of patients in their own communities. GPs experience cardiac arrest cases during the course of their daily work and provide prompt care which results in successful outcomes in urban, mixed and rural settings.

\section{INTRODUCTION}

Ireland's population of $4.5 \mathrm{~m}$ includes $38 \%$ who live in rural areas. ${ }^{1}$ Resuscitation outcomes from sudden cardiac death are

\section{ARTICLE SUMMARY}

Article focus

- Sudden cardiac death (SCD) is a leading cause of death in most countries; most events are related to ventricular fibrillation, caused by coronary artery disease. Cardiopulmonary resuscitation and defibrillation are the key interventions in dealing with SCD but are critically time-dependent.

- General practitioners (GP) are well-placed to deliver early care in many communities. However, the role of GPs in dealing with cardiac arrest in their communities has been little explored.

- 495 GPs joined this study over 5 years to report all cardiac arrests in which they participated in care.

Key messages

- Of the 272 cardiac arrests reported, the patient had a return of spontaneous circulation in $32 \%$ and was discharged from hospital in $18.7 \%$; these survival rates are higher than those generally reported for out-of-hospital cardiac arrest. Forty-nine patients survived cardiac arrest.

- A total of $36 \%$ of practices reported a cardiac arrest during the period; $13 \%$ had more than one event. Rural practices experienced twice as many events as urban/mixed practices but had similar survival rates.

- In $45 \%$ of cases, the first automated external defibrillator was brought by the GP and in $65 \%$ of cases, the GP was on the scene before the ambulance service.

Strengths and limitations of this study

- The 495 participating practices (about a quarter of Ireland's general practices) contributed 27000 months of defibrillator availability, with a mean $89 \%$ data collection rate.

- The duration, representativeness and good data collection rates of the study indicate that general practice has the potential to save additional lives in most communities.

- Caution is needed in generalising the experience of participating practices to all Irish practices and to other healthcare systems. 
traditionally poorer in rural areas, ${ }^{2-4}$ although recent Irish regional data suggest comparable urban and rural outcomes in one region. ${ }^{5}$ The Department of Health's report on sudden cardiac death estimated survival rates from out-of-hospital cardiac arrests in Ireland to be around $5 \%{ }^{6}$

This study reports the incidence and outcomes data for general practitioner (GP) involvement in cardiac arrest with resuscitation attempt (CARA) in urban, rural and mixed areas of Ireland over a 5-year period. Clinical data on the use of specific clinical interventions (eg, advanced life support measures) and ECG data will be reported separately. A CARA is a cardiac arrest in which GPs at the reporting practice have contributed to the resuscitation effort, whether alone or in conjunction with ambulance staff or other healthcare providers.

Early response to out-of-hospital cardiac arrest is critical to resuscitation, but not always possible. Solutions proposed include public access defibrillation, reduced emergency medical services call-response intervals and first responder schemes ${ }^{7-10}$; overall, a doubling of survival appears to result from early advisory external defibrillator (AED) use. $^{11}$ However, little research has explored the potential impact of GP delivered care in healthcare systems with well-established general practice structures. Available work suggests that general practice is well-placed to provide care and that GPs are willing to do so. ${ }^{12}{ }^{13}$ In 2009, initial data were reported from the Medical Emergency Responders Integration and Training (MERIT) Project in Ireland, indicating a discharge rate of $19.5 \%$ among 136 cardiac arrests managed by GPs. ${ }^{14}$

Ireland has a well-established general practice system delivered by around 2600 GPs, with significant involvement in local emergencies. ${ }^{15}$ This study describes 5-year data (2007-2011) for GP involvement in CARA in the community, with a specific focus on the experience of GPs in rural areas.

\section{MATERIALS AND METHODS}

Around 2600 GPs provide well-structured primary care in all areas of the country, organised in approximately 2000 general practices; around one-third of Irish GPs work in single-handed practice. ${ }^{16}$

The MERIT Project was established in 2006 to enhance the care of emergencies in the community. In 2006 and 2007, all 2347 GPs then identifiable in Ireland were contacted by letter and invited to join MERIT; 1361 (58\%) indicated interest, representing 811 centres of practice (table 1). Between 2006 and 2012, MERIT supported AEDs and immediate care training at 531 general practice sites in urban, rural and mixed areas. This study describes data collected by MERIT from the 495 sites participating in the 5-year study period 20072011, representing around one-quarter of Irish general practices.

\section{Table 1 Enrolment to MERIT}

2347 GPs identified in 2006 and invited to indicate interest (around 2000 centres of practice)

$136158 \%$ indicated interest (811 centres of practice)

531 General practices enrolled 2006-2013

495 General practices enrolled 2006-2011 (reported here)

GP, general practitioner

This is a prospective cross-sectional study. As GPs joined MERIT, they were immediately enrolled in the data collection process for all CARAs. Each quarter, a questionnaire survey was sent to the lead GP for the practice, asking whether or not a CARA had occurred in the previous 3 months; returns of these questionnaires over the 5-year period ranged from $81 \%$ to $97 \%$ of participating practices in each quarter, averaging $89 \%$. These returns also confirmed that practices had not experienced CARAs in the reporting period. Practices could report CARAs when they occurred-cross referencing ensured that no duplicates were reported in the quarterly survey.

When CARAs were reported, a nurse researcher carried out a structured interview with the GP, using a modified Utstein template including location, known patient of the practice, demographic data, times, interventions, rhythms identified, participating services, return of spontaneous circulation (ROSC) on scene or later, discharge from hospital and source of AEDs used. The modifications addressed compliance with local advanced life support (ALS) protocols, grades of the ambulance service practitioners involved and the patient's links with the practice.

The number of months in which a practice participated in MERIT during the 60-month-study period constituted 'AED months' and was calculated for each practice.

During the study period, the National Ambulance Service had no structured arrangements to notify GPs of cardiac arrests locally or to call on their assistance. Data were collected wherever possible on the source of the CARA.

Recruitment to MERIT involved at least one GP from the practice participating in a standard 1-day immediate care cardiac course, a skill-based course covering basic life support, AED use, standard prehospital advanced life support protocols based on the Irish national Clinical Practice Guidelines, ${ }^{17}$ teamworking and work with the ambulance services. Participants are recommended to retrain regularly and at least on a 2 yearly basis.

MERIT is funded by the Pre-Hospital Emergency Care Council, the Health Service Executive, the Department of Health and Health Research Board. MERIT worked closely with the North West Immediate Care Programme which fulfils similar roles, is funded by the Health 
Service Executive Western Region and supports defibrillator provision and training in that region. Many practices throughout the country have AEDs and staff members trained in basic and advanced life support; however, the MERIT Project offers standardised equipment, training, support and evaluation at the national level, which complements the work of individual practices.

Participating practices were asked to categorise their location as urban, rural or a mixture of both types of locations. There are no standardised definitions in use for urban, rural and mixed practice locations, but typically urban locations indicate the major cities of Dublin, Limerick, Cork, Galway, Waterford and Sligo; rural locations are small towns or villages in isolated or remote communities and mixed locations involve towns with rural hinterlands.

No patient-identifying information was collected. Missing data are reported (in text and tables below) in relation to cases where ascertainment of information could not be achieved; examples include the source of all AEDs used in cases or outcomes where the patient was unknown to the GP. Over the period, only partial data on outcomes were identified in 10 cases. ECG downloads are collated from any MERIT AED used in the resuscitation and are reported separately.

PASW Statistics 18 was used for data analysis. Descriptive statistics are reported for cumulative data. Univariate analysis was carried out to calculate unadjusted ORs ( $p$ values, CIs) for survival to discharge from hospital in relation to ROSC, witnessed collapse, rural versus other location, shock delivered and GP provided AED versus AED from other source. Ethics permission was received from the Research Ethics Committee of the Irish College of General Practitioners.

\section{RESULTS}

During the 60-month period, 495 practices in the MERIT network were almost equally divided among 168
(33.9\%) urban, $163(32.9 \%)$ rural and $164(33.1 \%)$ mixed sites. Cumulatively, AEDs were available for 27084 AED months and were available for equivalent periods in each of the three areas: urban areas 9108 AED months (33.6\%), rural areas 9252 AED months $(34.2 \%)$ and mixed areas 8724 AED months (32.2\%).

Table 2 reports 272 CARAs and summarises data over the 60 -month study period. In $45.2 \%$ of cases, the first AED on the scene was brought by the GP; in $14.7 \%$, the first AED was brought by a first responder, usually a layperson. In $65 \%$ of cases, the GP was on duty at the time of the incident and in 169/257 (65.8\%) cases was on the scene before the ambulance arrived.

Of all patients, $154(58.6 \%)$ were in a shockable rhythm at some point, ROSC was achieved in 87 (32\%) and $49(18.7 \%)$ survived to hospital discharge.

Witnessed arrests averaged $78.2 \% \quad(68.8-90.4 \%$ per year), $70.9 \%$ were male and median age was $60(0-89)$; no significant changes occurred in these characteristics over the period.

Table 3 summarises the involvement of general practices in CARAs, by region. Overall, 178 (36\%) practices were involved in at least one event during the 5-year period; $66(13.2 \%)$ were involved in more than one cardiac arrest and seven were involved in more than three incidents. It is striking that half of all rural practices were involved in at least one incident.

Tables 4 and 5 provide information on the relative involvement of practices in urban, rural or miscellaneous locations, by AED months and the number of CARAs reported. Table 4 summarises the ratio of CARAs to months of AED availability, by region. Rural practices (1 CARA per 74 months) are three times more likely to be involved in cardiac arrest management than urban practices (1 CARA per 240 months).

Table 5 presents data on CARAs by urban, rural or mixed location. It is striking that, in spite of the near equivalent number of available AED months in each

Table 2 Five-year CARA data, 2007-2011

\begin{tabular}{|c|c|c|c|c|c|c|}
\hline & 2007 & 2008 & 2009 & 2010 & 2011 & Total \\
\hline Cumulative AEDs & 360 & 426 & 455 & 471 & 495 & 495 \\
\hline Urban & 118 & 33 & 7 & 6 & 4 & $168(33.9 \%)$ \\
\hline Rural & 128 & 11 & 4 & 5 & 15 & $163(32.9 \%)$ \\
\hline Mixed & 114 & 22 & 18 & 5 & 5 & $164(33.1 \%)$ \\
\hline CARAs & 55 & 63 & 64 & 48 & 42 & 272 \\
\hline Shockable rhythm & 26 & 43 & 40 & 23 & 22 & $154 / 263(58.6 \%)$ \\
\hline ROSC & $14 / 55$ & $22 / 63$ & $25 / 64$ & $12 / 48$ & $14 / 42$ & $87 / 272(32.0 \%)$ \\
\hline Discharges & $10 / 53$ & $12 / 61$ & $14 / 60$ & $5 / 47$ & $8 / 41$ & 49/262 (18.7\%) \\
\hline Witnessed arrest & $41 / 5475.9 \%$ & $57 / 63(90.4 \%)$ & $50 / 64(78.1 \%)$ & $33 / 48(68.8 \%)$ & $31 / 42(73.8 \%)$ & $212 / 271(78.2 \%)$ \\
\hline GP on duty & $31 / 53(58.5 \%)$ & $43 / 63(68.3 \%)$ & $41 / 64(64 \%)$ & $30 / 48(62.5 \%)$ & $30 / 41$ (73.2\%) & $175 / 269(65 \%)$ \\
\hline \multicolumn{7}{|c|}{ Source of first AED $n=267$} \\
\hline GP & $32(58 \%)$ & 27 (42.9\%) & $21(32.8 \%)$ & 19 (39.6\%) & $24(57.1 \%)$ & $123(45.2 \%)$ \\
\hline Ambulance & 15 (27.3\%) & 25 (39.7\%) & $26(40.6 \%)$ & $23(47.9 \%)$ & 15 (35.7\%) & $104(38.2 \%)$ \\
\hline Lay & 8 & 10 & 15 & 5 & 2 & $40(14.7 \%)$ \\
\hline
\end{tabular}

AED, automated external defibrillators; CARA, cardiac arrest with resuscitation attempt; GP, general practitioner. 
Table 3 General practice involvement in a CARA, 2007-2011

\begin{tabular}{|c|c|c|c|c|}
\hline & $\begin{array}{l}\text { Urban } \\
168(33.9 \%)\end{array}$ & $\begin{array}{l}\text { Rural } \\
163^{\star}(32.9 \%)\end{array}$ & $\begin{array}{l}\text { Mixed } \\
164(33.1 \%)\end{array}$ & $\begin{array}{l}\text { Totals } \\
495\end{array}$ \\
\hline Not involved in a CARA & $133(78.6 \%)$ & $83(50.9 \%)$ & $101(61.6 \%)$ & $317(64 \%)$ \\
\hline Involved in a CARA & $35(20.8 \%)$ & $80(49.1 \%)$ & $63(38.4 \%)$ & $178(36 \%)$ \\
\hline CARAs $\times 1$ & 28 & 49 & 35 & $112(22.6 \%)$ \\
\hline CARAs $\times 2$ & 6 & 23 & 16 & $45(9.0 \%)$ \\
\hline CARAs $\times 3$ & 1 & 4 & 9 & $14(2.8 \%)$ \\
\hline CARAs $>3$ & 0 & 4 & 3 & $7(1.4 \%)$ \\
\hline
\end{tabular}

region, urban practices had significantly fewer events having participated in only $14 \%$ of all CARAs while rural practices contributed $46.3 \%$ and mixed practices $39.7 \%$.

The place at which the CARA occurred was reported in $269(98.9 \%)$ cases and was equivalent in urban, rural and mixed settings-CARA occurred in the GP practice in $39(14.4 \%)$ cases, the patient's home in $128(47.1 \%)$ cases and other locations in $102(37.9 \%)$ cases. No incidents are known to have been generated from ambulance control. Virtually all CARAs in the practice or patient's home were initiated by the patient or a friend or family member making contact with the GP.

There was no significant difference between survival to discharge rates in rural $(17.1 \%)$ and non-rural (20.1\%) settings (OR $0.9,95 \%$ CI 0.7 to 1.2 ). ORs were also calculated in relation to survival to hospital discharge for ROSC (OR 5.7, 95\% CI 4.2 to 7.7), witnessed collapse (OR 1.36, 95\% CI 1.3 to 1.5 ), defibrillation shock delivered (OR 1.8, 95\% CI 1.5 to 2.1), all of which indicate a positive contribution to survival. ORs for GP provided first AED on scene (vs all other sources) in relation to hospital discharge (OR 1.2, 95\% CI 0.9 to 1.6) did not show an improvement.

A fall in the numbers of CARAs reported by GPs has occurred in 2011 since a peak in 2009, particularly in urban areas.

\section{DISCUSSION}

This study is one of the first to provide systematic data on the experience of large numbers of GPs in the management of cardiac arrest in their communities. General practice involvement in CARAs in the community was sustained through the 5-year period of this study, with significant numbers of cases and successful outcomes. The outcomes of CARAs appear to be equivalent in urban, rural and mixed areas with a discharge rate from hospital of almost $19 \%$, with an average of 10 survivors reported annually. Henry et a $\bar{l}$ recently reported an out-of-hospital cardiac arrest (OHCA) discharge rate of $7.4 \%$ in one Irish region and the Sudden Cardiac Death Task Force Report estimated discharge rates nationally to be around $5 \% .{ }^{6}$ At $19 \%$, the discharge rates reported here suggest that a subgroup of patients are being identified and managed by GPs, perhaps involving more arrests witnessed by GPs. In almost two-thirds of our cases, the GP was on the scene before the arrival of the ambulance, suggesting that local availability is an important factor.

AEDs in rural practices are three times more likely to be used for cardiac arrest management than those in urban practices and half of all AEDs in rural areas have been used to manage a cardiac arrest in the 5-year period.

In $45 \%$ of all cases (and half of all rural cases), the first AED on the scene was brought by the GP involved, the GP was on the scene before the ambulance in $65 \%$ of cases and in $58.6 \%$ of all cases the patient had a shockable rhythm. Interestingly, $4 \%$ of practices were involved in the management of three or more CARAs in the period. These data strongly indicate that GPs are available at an early point in the cardiac arrest and that GPs in rural communities are a key resource in the management of emergencies in their communities.

Recent studies have highlighted lower survival in OHCA events occurring in the home in comparison to those in public areas. ${ }^{18} 19$ In this study, almost half of all cases occurred in the patient's home. The involvement of GPs may offer a mechanism to address the reported disadvantage generated in at-home events. Lay responders contributed the initial AED on the scene in $14 \%$ of cases, indicating a significant contribution to cardiac arrest management in the community. ${ }^{20}$ The ORs calculated here report similar determinants of survival to other studies in the area. The finding that survival is not influenced by the GP bringing the AED used, when compared with all other sources of an AED, is noteworthy and needs further investigation. The study is not structured or powered to identify a statistical difference between the GP provided AED, in comparison to other

Table 4 Cara incidence/AED months

\begin{tabular}{lllll}
\hline & Urban & Rural & Mixed & Overall \\
\hline CARAs & 38 & 126 & 108 & 272 \\
AED months & 9108 & 9252 & 8724 & 27084 \\
CARA/AED months & $1 / 240$ & $1 / 74$ & $1 / 81$ & $1 / 100$ \\
\hline
\end{tabular}

AED, automated external defibrillators; CARA, cardiac arrest with resuscitation attempt. 
Table 5 CARA by region, 2007-2011

\begin{tabular}{lllll}
\hline & Urban & Rural & Mixed & Totals \\
\hline CARA & $38(14 \%)$ & $126(46.3 \%)$ & $108(39.7 \%)$ & 272 \\
Shockable $(n=263)$ & $22 / 36(61.1 \%)$ & $68 / 123(55.3 \%)$ & $64 / 104(61.5 \%)$ & $154 / 263(58.6 \%)$ \\
ROSCs $(n=260)$ & $17 / 37(45.9 \%)$ & $31 / 121(25.6 \%)$ & $34 / 102(33.3 \%)$ & $82 / 260(31.5 \%)$ \\
Discharges $(n=262)$ & $5 / 34(14.7 \%)$ & $21 / 123(17.1 \%)$ & $23 / 105(21.9 \%)$ & $49 / 262(18.7 \%)$ \\
GP clinic/patient's home $(n=269)$ & $22 / 38(57.9 \%)$ & $80 / 123(65.0 \%)$ & $71 / 108(65.7 \%)$ & $173 / 269(64.3 \%)$ \\
Source of first AED $(n=267)$ & $17(44.7 \%)$ & $62(49.2 \%)$ & $44(40.7 \%)$ & $123(45.2 \%)$ \\
GP & $16(42.1 \%)$ & $42(33.3 \%)$ & $46(42.6 \%)$ & $104(38.2 \%)$ \\
Ambulance & 3 & 22 & 15 & $40(14.7 \%)$ \\
Lay & 3 & & \\
AED, automated external defibrillators; CARA, cardiac arrest with resuscitation attempt; GP, general practitioner; ROSC, return of \\
spontaneous circulation.
\end{tabular}

sources, but provides useful data to guide further hypothesis-driven research to address this question.

Berdowski et $a l^{21}$ have calculated a global mean incidence of emergency medical services treated adult OHCA of $62 / 10^{5}$ person-years, suggesting that Ireland experiences approximately 3000 such events annually. In a 2009 editorial, Ewy proposed that survival from out-of-hospital-cardiac arrest owing to a shockable rhythm should approach 40\%; where it does not, improvements in health services protocols should be expedited. ${ }^{22}$ We believe this study indicates that general practice may have a role in contributing to this goal, perhaps through mechanisms such as wider availability of AEDs and ALS training in general practice and closer links with the National Ambulance Service.

Our data show that MERIT GPs-around a quarter of Irish general practices-were involved in the active resuscitation of around 50 patients each year. Many Irish general practices outside the MERIT framework are also equipped with AEDs and may contribute to the management of incidents in their practices or communities. Our data suggest that the potential exists for GP involvement in 200-250 out-of-hospital cardiac arrests annually, if all GPs in Ireland were similarly involved, representing around $8 \%$ of all events in the country. With additional active links to the emergency medical services, further improvements might be achieved. Such protocol changes have significant potential both in Ireland and in countries with other well-established general practice systems.

\section{CONCLUSIONS}

Irish GPs encounter sudden cardiac death frequently, provide standardised care and have good outcomes. Most events occur within routine GP activities. These findings are similar in urban, rural and mixed areas, but rural GPs are more likely to be called on in OHCA than their urban counterparts. If all GPs in rural areas were equipped with AEDs, a significant additional capacity for early resuscitation would be available to those communities. If arrangements were in place for Ambulance Service control centres to alert those GPs to OHCAs in their communities, a further improvement in early responses might be achieved.

Acknowledgements The authors acknowledge the support of the Pre-Hospital Emergency Care Council, the Health Research Board, the Health Service Executive and the Department of Health. We thank the many general practitioners involved in this study who contributed their time and expertise to the care of patients and the collection of data.

Contributors GB is the Principal Investigator for the MERIT Project and has contributed to all components. $\mathrm{MH}$ and $\mathrm{ME}$ have designed and implemented all data collection, collation and analysis procedures. JD has contributed to the design, data collection and practice support throughout the period. All authors have contributed to the preparation of the manuscript.

Funding Pre-Hospital Emergency Care Council, Department of Health, Health Service Executive, Health Boards, Health Research Board.

\section{Competing interests None.}

Ethics approval Research Ethics Committee, Irish College of General Practitioners.

Provenance and peer review Not commissioned; externally peer reviewed. Data sharing statement No additional data are available.

\section{REFERENCES}

1. Census 2011. Town and country. Ireland: Central Statistics Office, 2012.

2. Sasson C, Rogers MA, Dahl J, et al. Predictors of survival from OHCA. Circ Cardiovasc Qual Outcomes 2010;3:63-81.

3. Yasunaga $\mathrm{H}$, Miyata $\mathrm{H}$, Horiguchi $\mathrm{H}$, et al. Population density, call-response interval and survival of out-of-hospital cardiac arrest. Int J Health Geogr 2011;10:26-35.

4. Jennings PA, Cameron P, Walker T, et al. Out of hospital cardiac arrest in Victoria: rural and urban outcomes. Med J Aust 2006;185:135-9.

5. Henry K, Murphy A, Wills D, et al. Out-of-hospital cardiac arrest in Cork, Ireland. Emerg Med J emermed-2011-200888. Published Online First: 15 June 2012.

6. Report of the Task Force on Sudden Cardiac Death. Dublin: Department of Health \& Children, 2006:88.

7. Moore MJ, Hamilton AJ, Cairns KJ, et al. The Northern Ireland Public Access Defibrillation (NIPAD) study: effectiveness in urban and rural populations. Heart 2008;94:1614-19.

8. Hallstrom A, Ornato JP. Public-access defibrillation and survival after out of hospital cardiac arrest. NEJM 2004;351:637-46.

9. Ciarns K, Hamilton AJJ, Marshall AH, et al. The obstacles to maximising the impact of public access defibrillation: an assessment of the dispatch mechanism for out of hospital cardiac arrest. Heart 2008;94:349-53.

10. Berdowski J, Blom MT, Bardai A, et al. Impact of onsite or dispatched AED use on survival after out-of-hospital cardiac arrest. Circulation 2011;124:2225-32.

11. Weisfeldt ML, Sitlani CM, Ornato JP, et al. Survival after application of AED before arrival of the emergency medical system: evaluation 
in the resuscitation outcomes consortium population of 21 million. $J$ Am Coll Cardiol 2010;55:1713-20.

12. Chialkias A, Koutsovasilis A, Mazarakis A, et al. Cardiac arrest in Greek primary health care and willingness of GPs to use AEDs. Resuscitation 2011;82:1144-7.

13. Ong ME, Yiong $\mathrm{HC}$, Hwee YA, et al. Resuscitation of out of hospital cardiac arrest by Asian primary care physicians. Resuscitation 2005;65:191-5.

14. Bury G, Headon M, Dixon M, et al. Cardiac arrest in Irish general practice: an observational study from 426 general practices. Resuscitation 2009;80:1244-7.

15. Bury G, Prunty H, Egan M, et al. Experience of pre-hospital emergency care among GPs in Ireland. EMJ 2008;25:450-4.

16. O'Dowd T, O'Kelly M, O'Kelly F. Structure of general practice in Ireland 1982-2005. Dublin: Irish College of General Practitioners, 2006.

17. Clinical Practice Guidelines, v2012 (3.2). Pre-Hospital Emergency Care Council, Ireland. http://www.phecit.ie/PHECC/
Clinical resources/Clinical_practice guidlines/2012 edition_CPGs/ PHECC/Exams_and_candidates/Clinical_Practice_Guidelines/ Clinical_Practice_Guidelines.aspx?Hke

18. Weisfeldt ML, Everson-Stewart S, Sitlani C, et al. Ventricular tachyarrhythmias after cardiac arrest in public versus at home. NEJM 2011;364:313-21.

19. Nakanishi N, Nishizawa S, Kitamura $Y$, et al. The increased mortality of witnessed out of hospital cardiac arrest occurring in the home. Prehosp Emerg Care 2011;15:271-7.

20. Community First Responder programmes. Pre-Hospital Emergency Care Council, Ireland. http://www.phecit.ie

21. Berdowski J, Berg RA, Tijssen JG, et al. Global incidences of out-of-hospital cardiac arrest and survival rates: systematic review of 67 prospective studies. Resuscitation 2010;81:1479-87.

22. Ewy GA. Do modifications of the American Heart Association guidelines improve survival for patients with out-of-hospital cardiac arrest? Circulation 2009;119:2542-4. 